\title{
LA PERSPECTIVA REGIONAL/LOCAL EN LA HISTORIOGRAFÍA SOCIAL ARGENTINA*
}

\author{
Regional perspective / local Social Historiographyc in Argentina
}

\section{Sandra Fernández**}

\section{Resumen}

El artículo pretende delinear un recorrido para abordar la historia regional/local argentina de los últimos años, deteniéndose especialmente en alguno de los hitos que hicieron posible su consolidación y proyección historiográfica. De este modo, se concentra en presentar de manera sucinta un balance de sus líneas de desarrollo, las influencias y aportes más destacadas, así como alguno de los debates y producciones realizadas desde el campo que impactaron en el derrotero de la historia regional y local argentina.

$$
<\text { Historia Regional }><\text { Historia Local }><\text { Argentina }>
$$

\begin{abstract}
The article seeks to outline a route in order to address the regional/local Argentine history over the last years, with special attention on the milestones that made possible its consolidation and historiographic projection. Hence, the article focuses on briefly presenting a balance of its lines of development, its most prominent influences and contributions, as well as some of the debates and productions carried out in this field of study which had an impact in the course of the regional and local history of Argentina.
\end{abstract}

$<$ Regional history $><$ Local History $><$ Argentina $>$

Recibido: 03/09/2015 // Aceptado: 06/11/2015

\footnotetext{
* Este artículo representa un balance de la conferencia "La perspectiva regional/local en la historiografía social argentina" realizada el 2/10/15 en la sede del Instituto de Investigaciones Geohistóricas (IIGHI), CONICET/UNNE en el marco de la presentación de la Especialización en Historia Regional, Secretaría de Investigación y Posgrado, Facultad de Humanidades- UNNE.

** CONICET/UNR.
} 
¡Cuánto es el esfuerzo que debemos realizar para legitimar un espacio de construcción historiográfica que de suyo tiene ya una larga tradición en la Argentina! Sin embargo tal esfuerzo, que puede ser mirado exclusivamente como un simple desgaste de energía, también puede presentarnos una contracara mucho más positiva. Si la primera opción puede invitarnos a pensar en lo innecesario del suceso, la segunda nos abre la puerta a la visión de la construcción de un espacio de encuentro y sistematización que genera un campo de acción muy dinámico dentro del horizonte de la historia nacional.

Dicho esto, la presentación de la perspectiva regional y local en la historiografía social argentina nos permite marcar algunos hitos que resultan importantes de resaltar. Muchas de las cosas que voy a enumerar ya fueron dichas en distintos trabajos previos, pero quizás este ejercicio sirva como una puesta en limpio de las reflexiones a las que debemos someter nuestras producciones. ${ }^{1}$

La cuestión regional/local puede ser comprendida y abarcada desde tres líneas claras de desarrollo. Más allá de la incumbencia tradicional de pensar sus alcances en base a su territorialidad, esta aproximación común como recorte temático tiene límites, literales y metafóricos, para proponer el efectivo análisis de un proceso histórico. Circunscribir un espacio desde un punto geográfico o administrativo no nos augura una buena investigación regional, simplemente nos señala a priori el efecto de uso de una dimensión espacial, que puede resultar o no pertinente. Adecuar de manera forzada un análisis a una realidad recortada geográfica o jurisdiccionalmente lejos de reconfortar el incentivo historiográfico puede relegarlo a la simple expresión de deseo de describir "lo cercano". ${ }^{2}$ Sin embargo pensar "lo cercano" responde también a un estímulo que con fuerte carga antropológica es capaz de comprender local y regionalmente lo que acontece. Dicho de otro modo observar e interpretar un problema en espacios reducidos permite obtener un nivel de interpretación que supera una mirada macro. De allí que según lo que nos interese preguntarnos, y sobre qué objetos pretendamos enfocarnos, la caja de herramientas utilizada responderá a una escala de análisis que guiará el estudio. La versatilidad del investigador hará el resto, sólo si es capaz de comprender que la investigación social va más allá de la simple aplicación de modelos.

La delimitación de la investigación regional/local en torno de un objeto de estudio ha sido un tópico también recurrente en las aproximaciones que se han desplegado desde los años sesenta del siglo pasado en adelante. Han sido habituales las afirmaciones que dicen algo así como "el objeto define la región". Ahora, si bien es cierto que el objeto tiene mucho que ver a la hora de reflexionar por lo regional/

1 Textos previos que pueden referenciar este contenido son Fernández (2013, 2008, 2007, 2006).

2 Los trabajos de este cariz si bien superan la idea de la delimitación institucional, en general pueden ahondar en una imagen perceptiva, que trabaja sobre la comprensión del territorio delimitándolo en un plano sensible. Estas formas de definición a partir de lo territorial involucran también otro nivel de complejidad y se encuentran ligadas a las condiciones de construcción de las identidades sociales. La condición de pertenencia o no a un "lugar", un adentro y un afuera marcado por los rasgos de identidad, el enraizamiento a un sitio, que hace referencia en muchos casos a lo propio, que dota de sentido a lo cercano son visiones que no dejan de representar una variable territorialista que no hace hincapié en lo formal o institucional sino que toma en como eje un concepto como la identidad y sus formas de percepción. 
local, también lo hace el contexto, el período, la cadena fenomenológica de eventos que permite que se pueda extraer una interpretación de lo real. El deseo de que el objeto de estudio defina la región, tiene sus peculiaridades. Una de ella es que el contenido otorgaría sentido al continente, lo que en términos de definiciones nos llevaría a una multiplicidad de eventos cargados de una fuerte carga simbólica. Desde esta perspectiva, una aproximación regional y local sería siempre distinta: objeto, período y espacio dan cuenta de realidades diferenciadas. El gesto generalizador se encontraría así reducido a la posibilidad cierta de un ejercicio comparativo, a partir del cual extraer afirmaciones comprobables. Si pretendemos explicar un proceso histórico en términos recortados, si le otorgamos la carga semántica al "estudio de caso", la estrategia es apropiada, y hasta podríamos afirmar que exitosa. Ahora bien si desde ese análisis pretendemos generalizar, las dificultades pueden llegar a ser abrumadoras. Es verdad también que muchas explicaciones centradas en este tipo de aproximaciones han resultado certeras a la hora de producir un conocimiento específico poniendo en tela de juicio interpretaciones previas o tradicionales sobre un tema instalado en la literatura historiográfica. Los ejemplos en estas últimas décadas son prolíficos y quizás las relecturas sobre la efectiva consolidación del estado Nacional sean de las más transitadas y más enriquecedoras. Sólo por señalar el proceso, y sin detenernos en su enumeración, los trabajos realizados sobre los antiguos territorios nacionales son representativos de tales motivaciones, y han redundado en corpus de significativa trascendencia, que puede rastrearse en las numerosas y prestigiosas publicaciones académicas del medio.

¿Qué es lo que hace distinta una aproximación regional a otra? Evidentemente el acercamiento metodológico y la idea de un contexto que otorga sentido y coherencia a las interpretaciones del fenómeno a estudiar. Esta matriz ha sido vastamente utilizada en, podríamos decir sin temor a equivocarnos, los últimos quince años. Sería extenso y complejo, en particular por las omisiones involuntarias que se puedan realizar, listar la larga producción que ha echado sus raíces en este modelo de investigación. Numerosos grupos de investigación en el campo, de norte a sur de la república, han permitido generar un caudal muy importante de nuevos resultados historiográficos qué han cambiado la forma en que varios de los problemas centrales de la historia argentina han sido tratados. Los ejemplos son varios. Si la historia económica fue capaz de capitalizar estos aportes de manera más rápida y eficiente, las líneas de trabajo propuestas sobre algunos temas nodales de la historiografía actual como la dictadura, o el primer peronismo hicieron lo suyo al incorporar de forma estable la producción derivada de la historia regional/local para pensar tales problemáticas fuera de las matrices de modelos con fuerte carga de interpretación sociológica y espacialmente circunscriptos a Buenos Aires, o en el mejor de los casos al área pampeana.

Las influencias han sido muchas, en particular de la historiografía europea. Es difícil establecer una frecuencia pero sí algunos tópicos sobre los que se puede hacer énfasis. En principio, la idea de la escala. Este artilugio metodológico se adapta muy bien a los análisis que rompen el paradigma del estado Nacional como horizonte omnipresente de la pesquisa. La frase hecha cuanto menor, mejor, dice mucho alrededor 
de la intensidad que la elección de la escala propone al momento llevar adelante la recopilación de la información, la formulación de hipótesis, y el proceso de interpretación y elaboración de resultados. Desde la más ingenua idea del microscopio, pasando por la metáfora de la red de pesca, hasta la más compleja concepción de Bernard Lepetit (2015) sobre la escala arquitectónica, los microhistoriadores europeos han influenciado mucho sobre el referente de la escala de tratamiento del problema tanto desde un plano metodológico como instrumental. ${ }^{3}$ Agudizar la mirada, poner el foco, concentrar la lente, han sido expresiones emanadas desde esta corriente para demarcar las formas de pensar el problema de estudio y de la delimitación de los corpus documentales. La microhistoria articula muy bien las dos primeras metáforas. ${ }^{4} \mathrm{Si}$ el microscopio, introduce la idea de la mirada intensa sobre lo que a simple vista no puede verse y reconocerse, la red lo hace en especial para imponer un recorte asociado a la cantidad, pertinencia y calidad de las fuentes a examinar. El primero entonces permite ver nuevos problemas sobre temas transitados: no estamos viendo sólo un trozo de piel, sino estamos observando las células que la componen. La red por su parte remite a la selección y clasificación de las fuentes. No es sólo lo que la malla de la red arrastra sino las dimensiones de la trama que sujeta o libera materiales en su interior. Una malla ajustada permite mayor cantidad de elementos susceptibles de análisis, pero también es incomoda al momento de procesar la información. La adecuada selección de las fuentes para el acercamiento historiográfico es el gesto metodológico esencial para llevar adelante la investigación tanto microhistórica como regional/local.

En simultáneo dos elementos más del escenario historiográfico europeo tuvieron una fuerte impronta sobre la historiografía regional/local. Acompañando las disquisiciones alrededor de la escala, la crítica del estado Nacional como único marco de referencia para la investigación dejó una huella durante los años setenta y ochenta al proyectar nuevos recorridos de investigación. La crisis del paradigma de la historia total hizo que en especial en España se agudizaran las miradas para interpretar realidades que habían estado por lo pronto opacadas en la historiografía dominante del momento. La historia contemporánea española, el período de la república, la dictadura franquista, y por sobre todo la recuperación de la historia de las comunidades, fue un impulso renovador que permitió un caudal de conocimiento, y una sugestiva búsqueda de nuevos objetos de estudio que eran abordados con metas de investigación más frescas. La historiografía española no sólo cuestionó profundamente la impronta que

\footnotetext{
3 Partiendo de que la microhistoria es incapaz de generalizar a partir de conocimiento producido en investigaciones realizadas a nivel local, Lepetit propone replantear el problema de la generalización a partir de la noción de escala. Tomada de la cartografía, esta noción se aplica a la historia para definir el conocimiento como un "modelo reducido" de la realidad. Esta visión propone la construcción de lo general desde lo particular, resituando entonces al individuo en el contexto, y dentro de la sociedad. Con lo cual también es posible ver lo macro en lo micro, desde y dentro de lo micro mismo, reubicando el caso en la norma y la norma actuando dentro del caso, etc. (Aguirre Rojas, 2004).

${ }^{4}$ Es una tarea que nos supera listar la impresionante bibliografía ligada a la microhistoria, sólo como recurso de síntesis pueden citarse dos textos que condensan muy bien las líneas de la corriente, Revel (2015) y Serna y Pons (2000), así como otros tres textos germinales de tal perspectiva historiográfica: Grendi (1996), Levi (1993), Ginzburg y Poni (1991).
} 
el annalismo había tenido sobre ella, sino que se permitió de manera más libre dialogar con tradiciones consolidadas, como el marxismo británico, y otras en pleno proceso de eclosión, como la voluble microhistoria italiana. Así el impacto de la historiografía local española sobre el ya significativo recorrido de la historia regional/local argentina, se manifestó de forma evidente durante los años noventa. Las posibilidades de intercambio académico de forma frecuente y fluida, hizo que el diálogo no sólo fuera a partir de lecturas bibliográficas, sino de un franco proceso de conocimiento mutuo a partir de eventos, programas, proyectos, etc. Los presupuestos vertidos por Casanova, Terradas, Serna y Pons, sintetizan los lineamientos generales de la historia local española, que más tributaron al espacio académico vernáculo. Todos ellos interpelan la idea del estado Nacional como objeto exclusivo del análisis histórico, pero lo más importante es que sugieren variables metodológicas de cambio para encarar los estudios regionales y locales no como referentes anecdóticos de un pasado más remoto o más cercano, ni tampoco como resultado de aproximaciones parciales, sino a partir de análisis exhaustivos de casos, capaces de otorgar representatividad para comprender horizontes mayores. La disyuntiva de la historiografía española en este sentido es que la aproximación regional/local no confirma procesos generales como reflejo de lo macro, sino a partir de la interpretación de lo específico, poniendo en cuestión las afirmaciones producidas desde la historia nacional. Si Casanova (1999) hace énfasis en la tensión metodológica alrededor de la generalización, y en la cuestión de la historia nacional como fórmula preponderante; Terradas (2001) por su parte pone el acento en la comprensión desde lo local y lo regional de lo que sucede en un nivel mayor, "a través de una sociedad, un país, una cultura, un mundo...”. Serna y Pons (2007), quizás oficiando casi como una síntesis al proponer una mirada más teórico-metodológica al asunto, señalan con mucha claridad que lo local y lo regional, en tanto categorías socialmente espacializadas, tienen importancia comprensiva, paradójicamente a partir de la conciencia de su artificialidad, y por lo tanto el peso de los conceptos se encuentra no sólo en un espacio físico, sino en el diseño de un tipo de investigación específica (historia regional y local). La meta, entonces, de toda investigación regional/local, para estos autores, no ha de ser sólo analizar la localidad, la comarca, la región, sino sobre todo estudiar determinados problemas en esos espacios, con un lenguaje y una perspectiva tales que la transposición del objeto implique una verdadera traducción, la superación del ámbito identitario.

En todas estas interpretaciones subyace una fuerte concepción de que para la Historia el contexto es sustancial; parte inherente de la disciplina, ajusta los niveles de observación en un plano epistemológico. Justamente la idea de contexto tan desarrollada en los estudios culturales británicos, fue uno de los elementos claves en la difusión e impacto de los resultados de la historiografía marxista en el medio europeo. Resume la tensión entre lo particular y lo general, pero por sobre todo toma materialidad en el juicio interpretativo sobre una cadena de eventos recortados sobre la diversidad, unidos en una coherencia fenomenológica mayor.

Estas influencias de índole fundamentalmente teóricas y metodológicas, si bien tuvieron una importante acogida en el medio local, impactaron sobre un campo que 
se estaba desarrollando de manera sistemática desde mediados de la década de 1960, porque en el origen de la historia regional y local argentina debemos colocar a Carlos Sempat Assadourian. Así es, los trabajos de Assadourian abrieron toda una línea de análisis que permitió comprender desde otra perspectiva las investigaciones regionales en la historiografía argentina. Sus estudios sobre la generación de mercados internos son un excelente ejemplo, y resultan una referencia insoslayable para cualquier abordaje historiográfico que pretenda encolumnarse dentro de los análisis regionales y locales.

Es este historiador cordobés quien introduce en las décadas de 1960 y 1970 una visión teórico-metodológica que marca una forma de tratamiento de problemáticas propias de la historia económico-social desde una perspectiva regional. Sus tempranos trabajos que se comenzaron a conocer aproximadamente a partir de mediados de la década de 1960, tendrían su corolario en su obra más difundida publicada en 1982: El sistema de la economía colonial. Mercado interno, regiones y espacio económico. La idea de espacio económico, que este autor delimitó en términos del análisis empírico, entendiendo a éste como un sistema de relaciones internas y externas que podían modificarse a lo largo de diferentes períodos históricos, habilitó un "complejo analítico" que si bien resaltaba que tal espacio estaba definido por la circulación de mercancías, también otorgaba coherencia al estudio de las relaciones políticas, económicas y sociales en él desplegadas. De este modo la región aparecía conceptualizada como un denso conjunto en el que se imbricaban elementos de signo diverso, como un sistema de articulaciones económicas, sociales, políticas y culturales. Hay que señalar además que las investigaciones de Assadourian superan por período y objeto de estudio la cuestión del estado Nacional. Propias del estudio de la organización colonial, sus interpretaciones ahondaban en la búsqueda de respuestas para pensar los elementos comunes, pero también los puntos disruptores en el proceso de la economía en los espacios americanos. Assadourian pensó en un complex en donde lo social y lo político convergían con lo económico; su mirada sin descartar una búsqueda explicativa mayor, optó además por una perspectiva más franca y horizontal que superaba lo macro. Su insistencia por la comprensión de lo regional abrió un horizonte de interpretación que nos sigue alcanzando medio siglo más tarde.

Tal impulso había sido posible merced a la influencia que en el medio sobre los años 1960s., había tenido la "renovación historiográfica". Se habían refrescado las lecturas, se había impuesto una transformación metodológica, y se había dado cuerpo a nuevas interpretaciones sobre temas recurrentes de la historia Nacional, entre ellas la cuestión del estado, marcado un punto de inflexión.

Los trabajos de Assadourian se recortan sobre las transformaciones que se imponían desde la segunda postguerra. Es que el clima de ideas impuesto por la historia social a nivel internacional permitió fructífero cruce de intereses y perspectivas en el campo. Con vertientes teóricas diversas la influencia de esta corriente llevó a que la producción dentro de la historia argentina se desplazara hacia nuevos rumbos, y que reflexionara con otra intensidad y crítica sobre nuestro pasado nacional. Dentro de los estudios regionales/locales, Assadourian representó la frescura de estas nuevas miradas, 
mixturada con las sólidas bases de la investigación de matriz colonial que tanto se había consolidado en nuestro medio. Así caminando a la par, los impulsos analíticos y generalizadores de los primeros años 1970s. tomaron varios de los supuestos generados diez o quince años antes para incorporar de manera creciente las hipótesis y líneas argumentales de este señero historiador.

Sin embargo, la dictadura, brutal en tantas formas y maneras, también lo fue para la producción científica, en especial dentro de las ciencias sociales. El movimiento y dinamismo de las investigaciones históricas, ya jakeadas en 1966, se detuvieron abruptamente, colapsados ante el quiebre social impuesto a comienzos de 1976. Tibiamente reaparecieron hacia 1982, luego del shock impuesto al régimen por la guerra de Malvinas, y producto de los cambios que se estaban desarrollando dentro de una sociedad oprimida, ávida de transformaciones. El reverdecimiento de foros de difusión y debate en distintos lugares del país, actuaron no sólo como palestras de divulgación y circulación de ideas y producciones sino también como ámbitos privilegiados de una sociabilidad quebrada años atrás.

Ahora bien si los trabajos de Assadourian representan una especie de génesis de la historia regional y local argentina, es en los años de la recuperación democrática en donde estos estudios se disparan en el horizonte de la investigación histórica. Reavivar el mejor impulso historiográfico, sofocado por la dictadura, hizo que las unidades académicas y los organismos de investigación retomaran la posta del camino truncado. El restablecimiento del gobierno democrático permitió volver a ocupar espacios universitarios, la normalización de organismos de investigación $\mathrm{y}$, sobre todo, hizo posible activar la investigación y la docencia académica, rescatando a colegas de exilios internos y externos, y permitiendo la rehabilitación del gesto historiográfico abierto en décadas anteriores. Más aún, a mediados de los años 1980s. se instalaron nuevas aproximaciones a problemas que habían quedado planteados pero no examinados y, por lo tanto, menos aún resueltos.

Tributarios de los trabajos de Assadourian fueron retomados por estos años los temas clásicos de la historia colonial, pero en especial los estudios alrededor de la función de Argentina en la división internacional del trabajo, e inmediatamente acerca de la formación del mercado interno; mercado interno que, como lo han demostrado las sucesivas e intensivas investigaciones, en la segunda mitad del siglo XIX distaba mucho de percibirse como nacional.

En este escenario reaparece la región como categoría susceptible de explicar procesos velados y vedados a análisis generales, marcando continuidades y diferencias; pero en especial cuestionando las bases sobre las que la generalización historiográfica se había construido. Si antes habían sido los circuitos mercantiles coloniales los protagonistas, ahora lo eran los estudios sobre la conformación de la clase dominante argentina. Las investigaciones realizadas desde distintos ámbitos académicos demostraron la ineficacia de exámenes exclusivamente centrados en la idea de "lo nacional". Concentradas en los estudios de otros espacios sociales argentinos 
(extrapampeanas y pampeanos) expusieron fenómenos de consolidación de grupos dominantes en distintas "regiones" del naciente Estado nacional.

Estas investigaciones con el marco explícito o implícito de la configuración y/o diferenciación regional proveyeron de un nuevo caudal de conocimientos, lo que conllevó a que se comenzara a tratar cada vez de forma más sistemática la cuestión de los alcances teórico-metodológicos de los estudios regionales y locales en historia.

Aquí la influencia de la historiografía europea fue predominante. Los debates y perspectivas que antes señalábamos se hicieron presentes en las discusiones y aproximaciones que la historia regional/local tuvo en el escenario argentino. Se convivió así, en este tiempo, con distintas formas de abordaje y diferentes praxis de investigación que oscilaron entre intensos estudios de caso, delimitaciones territorialistas, recortes y definiciones regionales, pero en especial se exploró nuevos objetos de estudio, y se buscó una mayor compensación teórica y metodológica a la hora de componer la investigación regional/local.

No estuvieron ausentes aquí también algunas lecturas sustanciales provenientes, en particular de la historia regional mexicana. Textos claves como Pueblo en Vilo de Luis González (1979), los artículos señeros de Eric Van Young (1987) y de Alan Knight (1998), así como los trabajos de Mario Cerutti (1985, 1987, 1989), hicieron que se prestara atención a la potencialidad de la perspectiva regional para intentar resolver problemas propios de la historia latinoamericana en su conjunto. ${ }^{5}$

Con todo este caudal de lecturas y experiencias, lo que se comenzó a evidenciar es que la denominación regional no proveía mecánicamente de una interpelación concreta del objeto de estudios, o una delimitación territorial; sino que lo que se pretendía en este nivel de estudio era una apuesta por diferenciar y definir un contexto de estudio, así como una decisión para pensar el diálogo entre lo particular y lo general en la Historia. Si antes habían sido los circuitos mercantiles coloniales los protagonistas, ahora lo eran los estudios sobre la conformación de la clase dominante argentina. Las investigaciones realizadas desde distintos ámbitos académicos demostraron la ineficacia de exámenes exclusivamente centrados en la idea de "lo nacional". Análisis concentrados en los estudios de otros espacios sociales argentinos (extrapampeanas y pampeanos) explicitaban fenómenos de consolidación de grupos dominantes en distintas "regiones" del naciente Estado nacional. Los efectos fueron sustanciales y se transfiguró la consideración que hasta el momento se tenía sobre la discusión y definición de la clase dominante argentina. ${ }^{6}$

La "primavera alfonsinista" reactivaba la investigación y la docencia en las universidades nacionales, pero en particular en organismos de investigación públicos.

\footnotetext{
5 Una buena síntesis sobre el particular puede encontrarse en el artículo de Hernán Vanegas (2012).

${ }^{6}$ En tanto tema central del desarrollo historiográfico argentino, numerosas investigaciones se concentraron en explorar las condiciones particulares de surgimiento de grupos dominantes locales, y en particular se detuvieron en el estudio de las formas de consolidación económica y social. La organización de redes, la capilaridad política entre grupos, la racionalidad económica, la distinción social fueron varios de los ejes por donde discurrieron los análisis, realizados en mayor o menor medida por distintos grupos e historiadores no radicados en Buenos Aires.
} 
Si bien breve, el impulso material y simbólico, permitió que se desplegaran nuevas líneas de análisis en el campo, pero fundamentalmente que se recuperara el trabajo colectivo, desarrollado ahora por colegas que venían de diferentes exilios, pero también por generaciones más jóvenes que encontraban en este diálogo la manera de saldar una deuda intelectual. Tales grupos de investigación tuvieron el mérito de iniciar una serie de proyectos de corte "regional" tendientes a profundizar y agudizar la mirada sobre problemas que hasta entonces habían sido evaluados como secundarios, o, mejor aún, ni siquiera habían aparecido en las miradas historiográficas. Varios espacios universitarios aparecieron como especialmente interesadas en este tipo de tópicos, con líneas de investigación interdependientes pero diferenciadas. Su marco de referencia fue el Estado nacional, pero cada uno de ellos se preocupó por hacer hincapié en los elementos que otorgaban "singularidad" regional a estos problemas encuadrados en la relación entre modernidad-Estado y/o clase dominante-mercado. Sería muy largo enumerar aquí la larga producción derivada de estos proyectos. Inicial a fines de los $80 \mathrm{~s}$, el conocimiento producido se consolidó durante los 90 s en un sinnúmero de artículos y colaboraciones, y ya en el cambio de siglo en libros de circulación nacional. ${ }^{7}$

En este contexto desde mediados de los 90s se comienza a observar el esfuerzo por discutir tales presupuestos. Hay varias pistas iniciales. En un principio se comenzó con paneles, mesas e intervenciones que pusieran en discusión el estatuto de los estudios regionales. Luego aparecieron los escritos más sistemáticos en revistas, y finalmente compilaciones dedicadas especialmente a su tratamiento. Sin embargo si un acontecimiento puede condensar el clima de debate es una reunión realizada a fines de los noventa.

En el año 1998 en el marco del I Simposio Argentino-Chileno de Historia Regional, organizado por la Universidad de la Patagonia Austral y el Consultado Chileno en Ushuaia, se dieron cita Susana Bandieri y José Carlos Chiaramonte. El contrapunto mostró dos posiciones, que de alguna manera resumían un clima de ideas alrededor de los estudios regionales y locales.

Bandieri con sus investigaciones centradas en una supuesta "área marginal" (la norpatagonia) para la comprensión de la constitución del Estado nacional argentino, cuestionaba las líneas fundamentales desarrolladas sobre la generación del mercado interno e internacional, al poner de manifiesto otra periodización para comprender este proceso, así como la explicitación de que el área investigada excedía el espacio nacional, rompiendo de este modo con la monolítica concepción de la región como integrada a un todo mayor y jerárquico. Recuperando, en términos de Assadourian, la idea de espacio

\footnotetext{
7 Arbitraria por cierto, es cualquier enumeración de la producción escrita desarrollada por estos largos años. Simplemente podemos decir que núcleos de investigación localizados en Tucumán, Rosario, Neuquén, Córdoba, Tandil, Luján, Santa Fe, Salta, Jujuy, Resistencia, Mendoza, Río Cuarto entre otros destinaron sus esfuerzos a propender un conocimiento más exhaustivo y contextual de la historia argentina contemporánea, haciendo especial referencia a la perspectiva regional/local de estas investigaciones. Tales investigaciones llevadas adelante en centros de investigación, unidades académicas, grupos y proyectos, en el marco de las universidades nacionales y CONICET, propició una divulgación.
} 
económico como un complex social, político y cultural, complejizaba el mecanicismo de la conformación del Estado nación durante el siglo XIX.

Bandieri no se detuvo allí y exploró además cuestiones inherentes a la definición conceptual de los estudios regionales y locales desde la práctica aportando un conocimiento sustancial al perfil historiográfico e interdisciplinar de la problemática. Sus investigaciones sobre la Patagonia en clave regional, hacían accequible pensar de otra manera a un ámbito territorial que usualmente se presumía ocupado social y económicamente desde un eje atlántico, como por mostrar un Estado nacional extremadamente exitoso en su penetración sobre los espacios hasta entonces dominados por los indígenas. Por otro lado introduce una delimitación de lo históricamente regional en relación a una preocupación persistente en torno de los cambios en la larga duración, en especial atendiendo al conocimiento de procesos sociales que comprenden y estructuran en un espacio y un tiempo determinados. Haciendo hincapié en la "reconstrucción de las relaciones entre los sujetos sociales que marcan la especificidad de sus manifestaciones", sus trabajos y los de su grupo de investigación comprenden que la variable espacial sea entendida como central en la conformación de las relaciones histórico-sociales. ${ }^{8}$

Por su lado Chiramonte fiel en los 90s a una concepción fuertemente política de la Historia, hacía énfasis en una visión epifenoménica de los estudios regionales. Así afirmaría en la reelaboración de su escrito de 1998, diez años después: "Si consideramos entonces que lo regional no es otra cosa que una modificación particular de fenómenos particulares -como los flujos comerciales o las vías de comunicación- observaremos entonces que nuestro real objetivo en la mayoría de los trabajos "regionales" no es la región sino aquellos fenómenos que tienen existencia real" (Chiaramonte, 2008).

Chiaramonte sin embargo reivindica la pertinencia de estos abordajes en tanto reacción a las miradas centralistas que habían dominado los estudios históricos, y critica acertadamente los trabajos "parroquilistas" trasvestidos en historia regional y local. Hay que considerar además que la historiografía argentina no se encontraba construida en estos años sobre una sólida base de estudios monográficos localizados, que permitiera tener un panorama vasto y extenso de la historia nacional como se produjo en Francia, mejor ejemplo de esta metodología. Por el contrario se tenían obras que si bien muy importantes, se habían organizado en torno de la delimitación de problemas intensamente tratados a partir de los cuales se producía una pertinente generalización.

Resumiendo lo que el debate Bandieri-Chiaramonte muestra es la preocupación al interior de la historiografía argentina por reconocer la importancia del status teórico metodológico transformador de los estudios regionales y locales. Lo que los 90s nos muestran es que la aproximación regional/local propone una intensa mirada sobre el objeto de análisis, y se concentra en el manejo y recorte de fuentes, y en la sistematización y análisis de datos; y que esa práctica lleva a una producción de conocimiento sustancial

\footnotetext{
8 Una condensación de las líneas fundamentales desplegadas por Susana Bandieri, pueden consultarse en sendos artículos de la autora de 2001 y 2007.
} 
para la transformación de base interpretativa de los fenómenos históricos tal como los conocíamos hasta entonces.

Ya en el siglo XXI, la historia regional/local se debate en la integración de sus tres variables más prolíficas en la producción argentina. La perspectiva del análisis de "lo cercano" con fuerte impulso antropológico, la recuperación del trazo assadouriano para explorar objetos de estudios plausibles para definir la región, y el redimensionamiento de las escalas de análisis. Estos tres ejes han sido y son caminos azarosos pero importantes para consolidar un corpus historiográfico que devela los ingentes esfuerzos desde nuestro campo para nutrir de más y mejores estudios a la Historia.

El anclaje exacerbado sobre la base territorial de la conceptualización de la región y de lo local, así como la imposición abrumadora dentro de los estudios históricos de la definición de estas categorías simplemente sobre la base de la exposición y el recorte de los objetos de estudio, han empañado el sustrato más interesante de la faceta renovadora de la historia social contemporánea, relegando o bien retardando la posibilidad de poder iniciar estudios comparativos de largo aliento que sienten las bases de un trabajo más delicado y profundo dentro de la investigación histórica nacional. La consecución de esa obra no es sólo un ejercicio de síntesis, sino también una prueba tanto de la incorporación de la vasta producción realizada desde los distintos enfoques provistos -los estudios regionales y locales- como de un esfuerzo de interpretación más abrumador y excitante que la simple y mecánica prolongación de resultados acotados a realidades ligadas al espacio bonaerense hacia el escenario nacional.

La exploración de los alcances de los estudios regionales, en especial sus preocupaciones teórico metodológicas han decantado en una atractiva producción, mucha de ella ligada a eventos académicos preocupados por esta línea. Mesas en las Jornadas Interescuelas, Encuentros dedicados específicamente a la temática, y obras colectivas que se han convertido en referencia para el tratamiento de la problemática en nuestra historiografía, han diseñado un estado del arte fundamental alrededor de las cuestiones regionales y locales. Así a la preocupación teórico-metodológica se le suma la incorporación de nuevos objetos de análisis, el tratamiento también novedoso de corpus documentales, y la proliferación de resultados que permiten por el momento complejizar la mirada sobre la trama histórica argentina, augurando la posibilidad de síntesis en el corto y mediano plazo.

En este camino la producción académica propuesta desde la historia regional/ local es el sustrato más importante en la historiografía argentina hoy para poder llevar adelante una aproximación a importantes análisis comparativos para la interpretación de nuestro pasado nacional.

Ahora bien qué significaría entonces hacer historia regional/local hoy en Argentina. Susana Bandieri en 1996 decía en un artículo precursor de este tipo de debates que el piso para pensar esta modalidad historiográfica era que fuera capaz de relacionar a los individuos y los grupos con las estructuras y los procesos sociales, y que sin apartarse de la teoría y el análisis contextual se desligara de la historia nacional como marco omnipresente de referencia. 
Es evidente que con este gran punto de referencia los estudios regionales y locales sólo pueden ser comprendidos en un marco historiográfico recostado en la historia social, en sus transformaciones e hitos; por lo que la disminución de la escala de observación no significa renunciar a los grandes temas sino a una atenta delimitación de fuentes, técnicas y método.

La insistencia sobre el contexto, que los estudios regionales y locales tienen, resulta fundamental para la Historia Social, porque se sitúa en las antípodas de la contemplación aislada del fragmento. De este modo los contextos y experiencias de los actores sólo pueden colocarse como eje de la reflexión a partir de localizar nuestras pretensiones explicativas, privilegiando la dimensión contextual como principio organizador de la investigación, y aquí la apelación a lo regional/local implica toda una declaración de principios, tanto teórica como metodológica, pues la reducción de escala no refiere meramente a un ajuste para ver lo que en un nivel macro no puede observarse, sino a un intento por revelar lo particular dentro de grandes procesos y fenómenos, que tiene interés en sí mismo, más allá de que pueda o no llegar a expresar tendencias generales. Así un abordaje natural de la matriz analítica, o como diría Grendi microanalítica, la historia regional/local se detiene en la recuperación de la experiencia de los sujetos, y por ello se convierte sugestivamente en una forma y una praxis potencialmente apta para abordar el estudio de movimientos sociales e identidades. Justamente, el sustento de la Historia se basa en la consideración de las relaciones interpersonales como sujeto histórico, y tal elección implica precisamente una decidida opción de escala. El resultado de esta correlación es que tales relaciones sociales están siempre estrechamente ligadas al espacio, al lugar, al territorio, esto es, a referentes tomados en alta consideración en la perspectiva regional/local.

Pasados ya casi cincuenta años de los trabajos pioneros de Assadourian, la historia regional y local, ha demostrado en su trayectoria que recupera su gesto historiográfico, concebido a partir de la claridad teórica y la praxis historiográfica, y lo traduce en el campo, para las nuevas generaciones de historiadores e historiadoras sociales argentinos, abriendo las puertas a un balance necesario, y a la proyección de un trabajo tanto de síntesis como de nuevas exploraciones, temáticas y teóricas. Buen pronóstico para capitalizar treinta años de producción académica en el campo de los estudios regionales y locales.

\section{Referencias bibliográficas}

Aguirre Rojas, C. 2003. "Invitación a otra microhistoria: la microhistoria italiana". Histórica, XXVII.2, 283-317.

Assadourian, C. S. 1982. El sistema de la economía colonial. Mercado interno, regiones y espacio económico. Lima: IEP.

Assadourian, C. S. 1991. "Integración y desintegración regional en el espacio colonial. Un enfoque histórico", En: Mercados e historia, México: Instituto Mora. 
Bandieri, S. 1996. "Entre lo micro y lo macro: la historia regional. Síntesis de una experiencia". Entrepasados, 11.

Bandieri, S. 2007. "Nuevas investigaciones, otra historia: la Patagonia en perspectiva regional”, en Fernández, S. (Comp.) Más allá del territorio. La historia regional y local como problema. Discusiones, balances y proyecciones. Rosario: Prohistoria Ediciones, pp. 47-72.

Bandieri, S. 2012. "La noción de espacio económico en Carlos Sempat Assadourian y sus posibilidades de uso en historias regionales de lugares y tiempos diferentes". Estudios del ISHIR, 4, Rosario, ISHIR/CONICET, pp. 27-42.

Casanova, J. 1999. "Historia Local, Historia Social y Microhistoria”. En: Rújula, P. y Peiró, I. (Coords.) La Historia Local en la España contemporánea. Barcelona: Universidad de Zaragoza, L’Avenc, pp. 17-28.

Cerutti, M. 1985. "Contribuciones recientes y relevancia de la investigación regional sobre la segunda parte del siglo XIX en México”. En: Boletín Americanista, 37, Barcelona, Universidad de Barcelona.

Cerutti, M. 1987. "El gran norte oriental y la formación del mercado nacional en México a finales del siglo XIX". Siglo XIX, 4, Monterrey, Instituto Mora.

Cerutti, M. 1989. Burguesía y Capitalismo en Monterrey. 1850-1910. México: Facultad de Filosofía y Letras, Universidad Autónoma de Nuevo León.

Chiaramonte, J. C. 2008. "Sobre el uso historiográfico del concepto de región”. Estudios sociales, 35, Santa Fe, Universidad Nacional del Litoral, 131-140.

Fernández, S. 2013. "Breve balance de la historiografía regional/local en Argentina". En: Andreucci, B., et. al., Espacio y regiones: diarios de viajes y relatos de inmigrantes. Luján: Universidad Nacional de Luján.

Fernández, S. 2008. "El revés de la trama: contexto y problemas de la historia regional y local". En: Bandieri, S.; Blanco, G. y Blanco, M. (Comp.) Las escalas de la Historia comparada. Tomo II: cuestiones regionales y estudios empresariales. Buenos Aires: Miño y Dávila. pp. 233-247.

Fernández, S. 2007. "Los estudios de historia Regional y Local: de la base territorial a la perspectiva teórico-metodológica”. En: Fernández, S. (comp.) Más allá del territorio. La historia regional y local como problema. Discusiones, balances y proyecciones. Rosario: Prohistoria Ediciones. pp. 31-46.

Fernández, S. 2006. "La historia sugerente. Los desafíos en la construcción de la historia regional y local". En: Mata de López, S. y Areces, N. (coord.) Historia Regional. Estudios de casos y reflexiones teóricas, Salta: EDUNSa., pp. 13-26.

Grendi, E. 1996. "Repensar la microhistoria". Entrepasados, 10, pp. 7-21.

Ginzburg, C. y Poni, C. 1991. El nombre y el cómo: intercambio desigual y mercado historiográfico. Historia Social, 10, pp. 63-70.

González, L. 1979. Pueblo en vilo, Microhistoria de San José de Gracia. México: E1 colegio de México.

Knight, A. 1998. "Latinoamérica un balance historiográfico”. Historia y Grafía, 10.

Lepetit, B. 2015. "De la escala en la Historia". En: Revel, J. Juego de Escalas: experiencias de microanálisis. San Martín: UNSAM Edita, pp. 87-114. 
Levi, G. 1993. "Sobre Microhistoria". En: Burke, Peter (Comp.) Formas de hacer historia, Madrid: Alianza Universidad.

Revel, J. 2015. Juego de Escalas: experiencias de microanálisis. San Martín: UNSAM Edita.

Serna, J. y Pons, A. 2000. Cómo se escribe la microhistoria. Madrid: Cátedra.

Serna, J. y Pons, A. 2007. "Más cerca, más denso. La historia local y sus metáforas". En: Fernández, S. (Comp.) Más allá del territorio. La historia regional y local como problema. Discusiones, balances y proyecciones, Rosario: Prohistoria Ediciones, pp. 17-31.

Terradas, I. 2001. "La historia de las estructuras y la historia de la vida. Reflexiones sobre las formas de relacionar la historia local y la historia general". En: Fernández, S. y Dalla Corte, G. (comps.). Lugares para la Historia. Espacio, Historia Regional e Historia Local en los Estudios Contemporáneos. Rosario: UNR Editora, pp. 179-208.

Van Young, E. 1987. "Haciendo historia regional. Consideraciones metodológicas y teóricas". Anuario IEHS, 2, UNICEN.

Vanegas Delgado, H. 2012. "La región en su perspectiva histórica". Estudios del ISHIR, 4, Rosario, ISHIR/CONICET, pp. 4-26. 